\title{
BMJ Open Developing the Network Pain Rehabilitation Limburg: a feasibility study protocol
}

\author{
Cynthia Lamper, ${ }^{\oplus 1}$ Mariëlle Kroese, ${ }^{2}$ Albère Köke, ${ }^{1,3}$ Dirk Ruwaard, ${ }^{2}$ \\ Jeanine Verbunt, ${ }^{1,3}$ Ivan Huijnen ${ }^{1,3}$
}

To cite: Lamper C, Kroese M, Köke A, et al. Developing the Network Pain Rehabilitation Limburg: a feasibility study protocol. BMJ Open 2019;9:e025962. doi:10.1136/ bmjopen-2018-025962

- Prepublication history for this paper is available online To view these files please visit the journal online (http://dx.doi. org/10.1136/bmjopen-2018025962).

Received 10 August 2018 Revised 22 May 2019 Accepted 10 June 2019

\section{Check for updates}

\section{Author(s) (or their} employer(s)) 2019. Re-use permitted under CC BY-NC. No commercial re-use. See rights and permissions. Published by BMJ.

${ }^{1}$ Department of Rehabilitation Medicine, Maastricht University CAPHRI School for Public Health and Primary Care, Maastricht, The Netherlands

${ }^{2}$ Department of Health Services Research, Maastricht University CAPHRI School for Public Health and Primary Care, Maastricht, The Netherlands

${ }^{3}$ Kenniscentrum, Adelante, Hoensbroek, The Netherlands

Correspondence to Cynthia Lamper; cynthia.lamper@ maastrichtuniversity.nl

\section{ABSTRACT}

Introduction Patients having chronic musculoskeletal pain (CMP) face challenges as mismatches often exist between the complexity of patient's pain problem and the rehabilitation treatment offered. This can result in less efficient care for the patient and increased medical shopping. The Network Pain Rehabilitation Limburg (NPRL), a transmural integrated healthcare network, will be designed to improve daily care for patients with CMP. NPRL focusses on improving patient's level of functioning despite pain by stimulating a biopsychosocial approach given by all involved healthcare professionals. A feasibility study will be performed which will give insight into the barriers and facilitators, perceived value, acceptability and implementation strategies for NPRL.

Methods and analysis This study has a three-phase iterative and incremental design, based on key principles of a user-centred design. Mixed methods will be used in which healthcare professionals and patients involved in NPRL will participate. In phase 1, NPRL will be developed and healthcare professionals educated. Phase 2 focusses on the implementation and phase 3 on the transferability of NPRL. In addition, preliminary data on patient's work status, general health and participation level will be collected. The qualitative results of each phase will be analysed following the Consolidated Framework for Implementation Research (CFIR) and will be used to refine NPRL in daily practise.

Ethics and dissemination Informed consent will be obtained from all participants. The results of this feasibility study will form the basis for refinement of NPRL and planning of a large-scale process and effect evaluation of the Quadruple Aim outcomes. Dissemination will include publications and presentations at national and international conferences. Ethical approval for this study was granted by the Medical Ethics Committee Z, the Netherlands, METC $17 \mathrm{~N}-133$.

\section{INTRODUCTION}

Nineteen per cent of adults in Europe suffer from moderate to severe chronic pain with a duration of at least 6 months according to a large-scale epidemiological study. ${ }^{1}$ Also, about $18 \%$ of adults in the Netherlands have moderate to severe general chronic pain. ${ }^{2}$ Almost $90 \%$ of individuals with chronic pain

\section{Strengths and limitations of this study}

This study will be the first to evaluate feasibility of a transmural network for pain patient's rehabilitation, which provides integrated care aiming to improve their level of functioning despite pain by stimulating a biopsychosocial approach given by all involved healthcare professionals.

- In an iterative, user-centred design, mixed methods will be used to evaluate the barriers and facilitators, perceived value, acceptability and implementation strategies for the development, implementation and transferability.

- Data will be analysed using the Consolidated Framework for Implementation Research by Damschroder et al.

- The evidence generated from this feasibility study will not only help to adjust the design and content of Network Pain Rehabilitation Limburg, but will also help future studies with developing and implementing transmural networks in healthcare.

- Depending on the results of this feasibility study, a large-scale process and effect evaluation on the Quadruple Aim outcomes will be planned.

had experienced it for over 2 years. ${ }^{3}$ The most reported chronic pain complaint was chronic musculoskeletal pain (CMP). CMP is a complex biopsychosocial experience that varies widely between people depending on the context and meaning of the pain and the impact of psychosocial factors on patient's functioning. ${ }^{45}$

Breivik et $a l^{1}$ found that people with CMP were less able or even unable to do a range of daily activities and to maintain an independent lifestyle. In addition to pain itself, patients with CMP are often confronted with an elevated level of disability, depression and anxiety resulting in an increased disease burden. $^{6-8}$ In addition, work absenteeism among these patients is very high. ${ }^{910}$ In recent years, the direct and indirect costs for patients with CMP are estimated at 20 billion euros in the Netherlands. ${ }^{11}$ These costs 
are even higher than the annual costs of heart disease, cancer and diabetes. ${ }^{12}$ Although costs for CMP are high, only up to $60 \%-74 \%$ of patients with CMP get treated, and only $2 \%-5 \%$ get treated by a pain management specialist. ${ }^{1213}$ Currently, regardless of the treatment received, 34\%-79\% of Dutch patients with CMP still indicate a feeling of inadequate treatment. ${ }^{2} 14$ These patients seek a diagnosis or solution to their pain problem, which explains medical shopping. Even $61 \%$ of patients that started a multidisciplinary rehabilitation programme visited 6 to $>20$ different healthcare professionals 1 year before starting with multidisciplinary rehabilitation programme. ${ }^{15}$ A potential reason for these inefficiencies might be that the complexity of the patient's pain problem does not match with treatment as delivered, resulting in overtreatment or undertreatment, ${ }^{16}$ which highlights the need for adequate (cost) effective treatment strategies.

This mismatch may be explained by the fact that the knowledge and perspective of healthcare professionals, decision makers and the public varies regarding CMP, referral and treatment. ${ }^{13}$ Healthcare professionals receive inadequate training on the diagnosis and treatment of CMP, causing different points of view. ${ }^{17}$ Some healthcare professionals are more biomedical oriented and focus on explaining and solving the pain, whereas others are more biopsychosocial oriented and focus on optimising functioning despite CMP. ${ }^{18}$ Therefore, referral and treatment selections vary among healthcare professionals, which may result in less efficient care for patients with CMP.

Besides the different perspectives regarding CMP, general practitioners (GPs) in primary care and rehabilitation physicians (RPs) in secondary and tertiary care refer patients mostly based on their anamnesis and clinical experience. However, it appears to be difficult for GPs to identify the impact of all psychosocial factors on patients with chronic low back pain, one of the most frequently encountered CMP problems. ${ }^{19}$ Recently, different tools became available to support GPs in the decision-making process concerning (initial) treatment options for patients with chronic low back pain and fibromyalgia, especially focusing on the impact of psychosocial components. ${ }^{20-23}$ However, these decision-making tools are not implemented in daily care yet in the Netherlands. In the Dutch healthcare system, patients with moderate to severe levels of disability and associated influencing psychosocial factors are seen by an RP. To support decision making by RPs, an evidence-based objective tool to classify patients objectively and transparently for a specific treatment is needed. Earlier studies have shown that the inter-rater reliability of the method currently used by RPs to classify the level of disability (WPN classification) is at least questionable. ${ }^{24}{ }^{25}$ In addition, healthcare professionals indicate a lack of overview regarding the complete supply of treatment methods, resulting in inadequate referrals. ${ }^{26}$

Ideally, after assessing the level of disability, the patient receives a treatment matching the complexity of the pain problem in line with the biopsychosocial profile. As in most situations, no cure for CMP is possible and evidence-based treatments are multicomponent pain rehabilitation with a biopsychosocial focus on being active and living a valuable life despite pain. ${ }^{57-29}$ In primary care physiotherapy, cognitive-behavioural interventions and interventions focusing on biopsychosocial factors have shown long-term effects on patient outcomes. ${ }^{30} 31$ Moreover, even positive effects were found when advice combined with pain education alone is given by GPs or therapists to patients with $\mathrm{CMP}^{32-34}$ In secondary and tertiary care, multidisciplinary pain rehabilitation programmes with physical, psychological and/or social/ work-related components, like Acceptance and Commitment Therapy (ACT), Graded Activity (GA) and Exposure in vivo (EXP), are more effective than treatments focusing on one aspect of the biopsychosocial model for decreasing pain and disability in patients with disabling chronic low back pain. ${ }^{35-40}$

Despite this knowledge of the effective components of multidisciplinary rehabilitation programmes, a wide variety of treatment approaches in various dosages are currently applied in regular rehabilitation programmes in different private and public rehabilitation centres. ${ }^{41}$ To overcome the different points of view as well as the lack of overview about treatment options, objective decision-making tools and variety of treatments in the Netherlands, a national care standard for chronic pain was presented in 2017. ${ }^{11}$ In this standard, a matched and person-centred care approach for patients with CMP was proposed. ${ }^{42}$

To implement care as part of the national care standard, a transmural network could be designed in which different healthcare professionals collaborate in providing person-centred rehabilitation care. Recently, different transmural integrated care health networks, for example, for Parkinson's disease and palliative care, have been successfully developed and implemented in the Netherlands. ${ }^{43}{ }^{44}$ In line with these findings, a transmural pain rehabilitation network can provide a shared vision regarding CMP. It will include early recognition of patients with subacute pain followed by suitable person-centred treatment and referral. The treatment within the network is supposed to improve patients' levels of functioning despite pain and to prevent medical shopping of patients with CMP. ${ }^{11}$ It should have an unambiguous view, matched care and a person-centred approach with guidelines for referral and treatment, coordination and a continuous focus on improvement of care to increase the effectiveness, quality and efficiency of healthcare for patients with CMP. ${ }^{45}$ This approach fits with the advice of the WHO to focus on stimulating functioning when designing rehabilitation care. ${ }^{46} 47$

The Network Pain Rehabilitation Limburg (NPRL), a transmural healthcare network for CMP rehabilitation, will be designed to ultimately fulfil the Quadruple Aim in the province of Limburg, the Netherlands. ${ }^{48}{ }^{49}$ NPRL provides integrated care for patients with CMP in order to improve their level of functioning despite pain by stimulating a biopsychosocial approach for all involved 


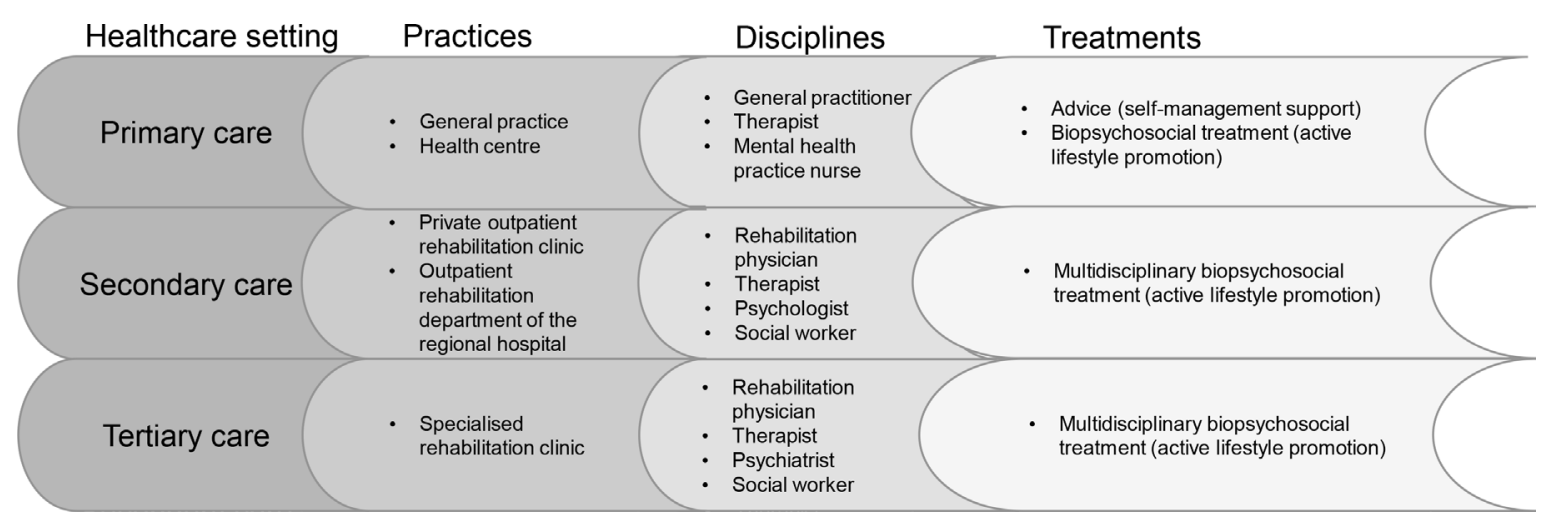

Figure 1 Construction of the healthcare system in Network Pain Rehabilitation Limburg.

healthcare professionals. As a first step, a feasibility study will be performed. This study aims to provide insight into the barriers and facilitators, perceived value, acceptability and implementation strategies for the development, implementation and transferability of the NPRL. This paper describes the study protocol of the feasibility study of NPRL for adults with CMP.

\section{METHODS}

\section{Study design}

A feasibility study with an iterative and incremental design, based on key principles of user-centred design ${ }^{50} 51$ will be conducted in the South-East region of the Netherlands from October 2017 until October 2018. This will follow the UK Medical Research Council framework ${ }^{52}$ for developing complex interventions. It will be useful as NPRL is a complex intervention because of the number of practices and integrated healthcare settings targeted in the NPRL and the number and variability of outcomes. In this iterative process, the development of NPRL will take place in three phases, namely development, implementation and transferability. The results of each phase will be used to refine the elements of the intervention and to shape the next phase, in which the barriers and facilitators of the different phases will be evaluated. During meetings, all healthcare professionals involved will be informed about the results and the adjustments to NPRL. In the subsequent phase, new adjustments will be integrated in daily practise. The development and implementation process will be 'practise-focused', indicating that the development will be based on the healthcare professionals' experiences with the current healthcare situation.

In phase 1, exploration of context will take place in order to develop the design of the NPRL and to educate the healthcare professionals involved. The focus will be on the barriers and facilitators in the development process of NPRL. Next, in phase 2 (implementation), the project focus will be on the specification of the content to adjust the design of the NPRL to daily practice. More insight into the barriers and facilitators of the implementation process will be collected. In phase 3 (transferability), the project will focus on the organisation of care in daily practise and the research focus will be on the barriers and facilitators for further implementation in other practices and organisations. In addition, preliminary data on efficiency will be collected. The qualitative data collected during the study will be analysed using the Consolidated Framework for Implementation Research (CFIR).$^{53}$ NPRL will be feasible in daily practise if the studied barriers and facilitators from the perspectives of healthcare professionals and patients are translatable to policies or guidelines that can be adjusted and integrated in daily practise.

\section{Participants}

In this transmural NPRL, healthcare professionals from different disciplines (GPs, physiotherapists, exercise therapists, mental health practice nurses, RPs and rehabilitation teams) and different healthcare settings (primary, secondary and tertiary care) will be asked for participation (figure 1). The setting in primary care concerns general and therapy practices, in secondary care a private outpatient rehabilitation clinic and the outpatient rehabilitation department of a regional hospital and in tertiary care a specialised rehabilitation clinic. The quality criteria established for practices and organisations for enrolling in NPRL are described in table 1.

In primary care, the recruitment will start with a primary care therapist or a GP interested in pain, and after consent to participate in NPRL. This person will be asked to recruit a GP or therapist with whom they already have intensive collaboration. For secondary and tertiary care, main organisations in the region providing rehabilitation care for patients with CMP will be asked to participate, so all healthcare settings in this region will be covered. Because of the nature and aim of this feasibility study, we decided to keep the number of healthcare professionals restricted. Based on earlier research, it has to be expected that, in this situation, the implementation process in daily practise can be easily adjusted when barriers arise. ${ }^{44}$

In addition to the involvement of healthcare professionals in this study, all patients treated by the participating healthcare professionals will be asked to evaluate NPRL and the perceived quality of care. The inclusion criteria for patients to participate in this study are described in table 2. 
Table 1 Inclusion criteria for healthcare professionals for enrolling in NPRL

$\begin{array}{ll}\text { Inclusion } & \text { Exclusion } \\ \text { Having a practice in the pilot area of NPRL. } & \begin{array}{l}\text { A GP who has visited less than } 2 \text { out of } 3 \text { education days } \\ \text { or a therapist who has participated in less than } 3 \text { out of } 4 \\ \text { education days. } \\ \text { different elements of NPRL. }\end{array} \\ \text { GPs and mental health practice nurses must be linked to } & \begin{array}{l}\text { Are not able to implement the protocols or assessment tool } \\ \text { of NPRL in their own practice. }\end{array} \\ \text { a participating therapist in order to make effective referrals } & \\ \text { to treat patients in (interdisciplinary) primary care regarding } & \\ \text { the protocol and vision of NPRL. } & \\ \text { Physiotherapists having a participating GP or RP. As they } \\ \text { cannot refer a patient when the patient is too complex } \\ \text { for them, they will not have an inclusion option for study } \\ \text { participants if there is no participating GP or RP. } \\ \text { Secondary and tertiary organisations have to meet } \\ \text { the criteria of the Position Paper 'Medical Specialist } \\ \text { Rehabilitation for chronic musculoskeletal pain' [2017]. }\end{array}$

GP, general practitioner; NPRL, Network Pain Rehabilitation Limburg; RP, rehabilitation physician.

It is expected that approximately 100 patients from all participating healthcare settings will give informed consent during the course of this study. They will receive questionnaires regarding satisfaction with care and their health status and pain-related disability. Moreover, a sample of approximately 10 patients, who finished a treatment according to the protocol of NPRL, will be recruited for a focus group. In this focus group, more information about barriers and facilitators from a patient perspective will be collected. In this way, patients are able to react to each other which will illuminate various perspectives which leads to a faster data saturation about each topic, which is an advantage above interviews. ${ }^{54}$

\section{Intervention: NPRL}

The main aim of NPRL is to provide integrated care for patients with CMP in order to improve their level of functioning despite pain by stimulating a biopsychosocial approach for all involved healthcare professionals. This should accomplish the Quadruple Aim: improvement of CMP patient functioning, experiences of care and worklife satisfaction of physicians and staff, as well as a reduction of healthcare costs of patients with CMP.
Each patient will receive the treatment needed to reach his/her optimal level of functioning. In order to reach this, a matched care approach will be used for every individual patient. Depending on the level of disability and biopsychosocial factors involved, this will either include (1) education only and no further treatment, (2) monodisciplinary treatment in primary care, (3) multidisciplinary treatment in primary care (collaboration between GPs, primary care therapists and mental health practice nurses in assessing and treating patients with CMP who need mental support besides physical exercise), (4) interdisciplinary treatment in secondary or (5) interdisciplinary treatment in tertiary care. Collaboration will be supported by facilitating communication between patients and all healthcare professionals involved in the trajectory of an individual patient by E-health. ${ }^{55}$ In addition, the collaboration between healthcare professionals in different practices and organisations will be further supported by informative meetings and education days. All healthcare professionals with different specialisms will participate together in the meetings and education days. This ensures a common understanding of the

Table 2 Inclusion criteria for patients in this feasibility study

$\begin{array}{ll}\text { Inclusion } & \text { Exclusion } \\ \begin{array}{l}\text { Age } \geq 18 \text { years old at the start of the study. } \\ \text { Patient living in the pilot area (physiotherapist, GP or RP) of }\end{array} & \begin{array}{l}\text { Any suspicion of a medical (orthopaedic, rheumatic or } \\ \text { neurological) disease that can explain the current pain (eg, } \\ \text { NPRL. }\end{array} \\ \begin{array}{ll}\text { Having musculoskeletal pain that is (suspected to be) } \\ \text { chronic. }\end{array} & \begin{array}{l}\text { Any suspicient therapy. } \\ \text { Treatment aim of the patient is to improve functioning }\end{array} \\ \begin{array}{l}\text { despite the pain. } \\ \text { Adequate Dutch literacy to complete the assessments. }\end{array} & \text { the expert opinion of the GP and RP. }\end{array}$

GP, general practitioner; NPRL, Network Pain Rehabilitation Limburg; RP, rehabilitation physician. 


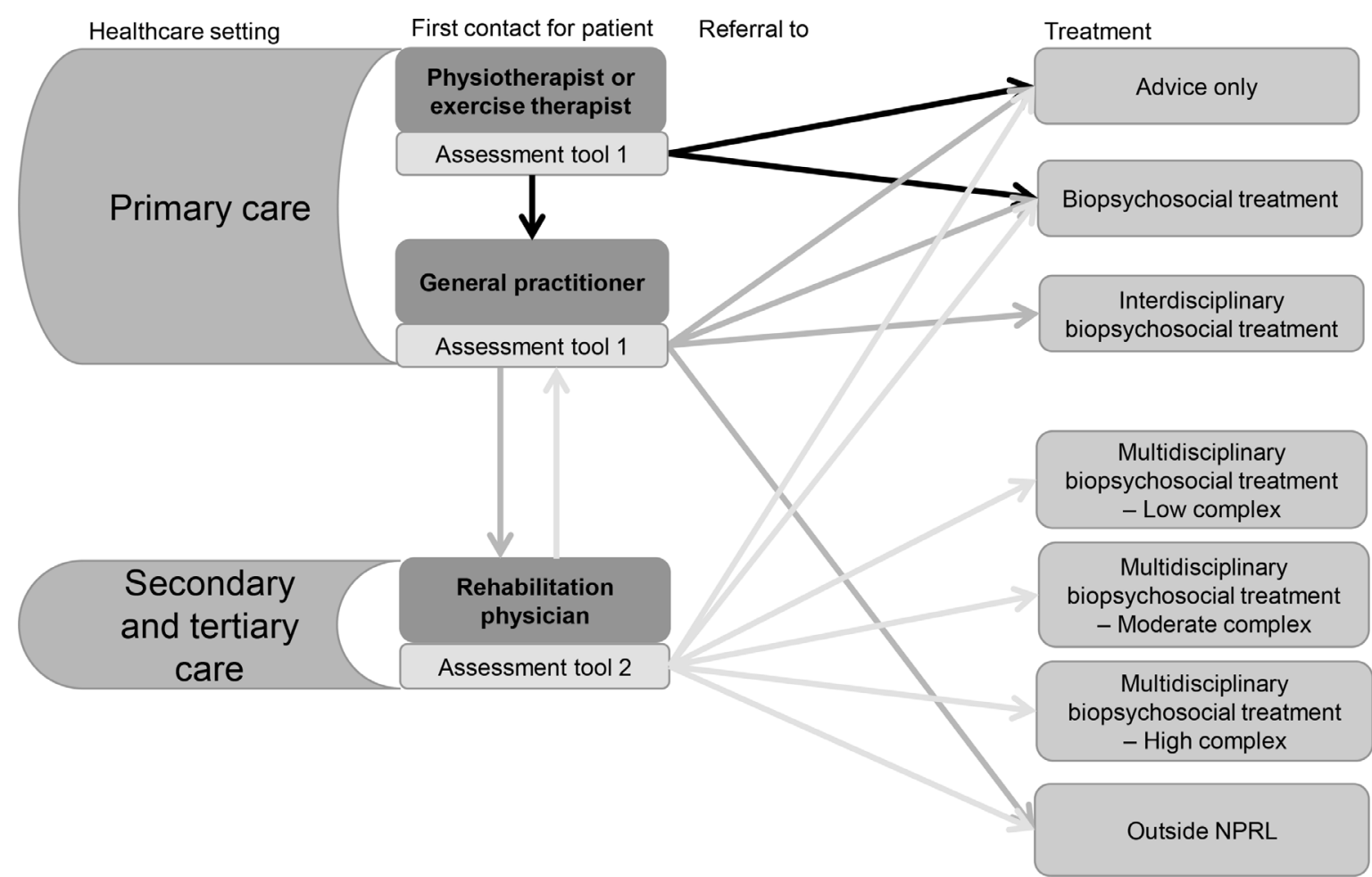

Figure 2 First patient contact and referral options per healthcare setting and discipline. NPRL, Network Pain Rehabilitation Limburg.

biopsychosocial approach and rehabilitation treatment options. In order to facilitate this in daily practice, the following elements are integrated in NPRL.

\section{Integral focus on assessment and referral: assessment tools}

To support the healthcare professionals in their decision making for problem mapping and treatment selection, two evidence-based objective assessment tools will be used. These tools will support the assessment of the complexity of the pain problem; one tool for GPs and primary care therapists and one tool for RPs. The assessment tool for primary care is based on the Start Back Tool ${ }^{20}$ and will help to advise patient treatment matched to the patient's biopsychosocial profile. The options are: advice only, treatments in primary care or for decision making by a RP (figure 2). The GP can also decide to advise patients for a treatment outside NPRL (psychiatrist, specific healthcare specialist, etc). Since 2006, patients in the Netherlands can visit a primary care therapist without a referral of a GP, ${ }^{56}$ so these therapists will also use this assessment tool. In this situation, a primary care therapist of NPRL will advise the patient to visit the GP for additional assessment and referral if needed as the GP is the gatekeeper to secondary and tertiary care.

When a patient visits an RP, the assessment tool for specialised rehabilitation care is used for decision making. This tool will assess the patient's view as well as the RP's view of the biopsychosocial problem and consists of two parts. The first part will guide the anamnesis of the RP and is based on two different ways to score disability-related complexity, namely the Case Complexity Index and
INTERMED method..$^{25} 5758$ First, a standardised scoring method for assessing the biopsychosocial profile and care for the past and current situations will be used by the RP. Second, a set of CMP-related questionnaires assessing anxiety, depression, catastrophising, fatigue, pain level, participation level and general health will be completed by the patient. After completion of these questionnaires, scores will be interpreted by the RP. Based on scoring in both parts of the RP assessment tool, patients will be categorised by profile, representing the patient's level of disability. In addition to primary and interdisciplinary primary care, the second tool will assist the RP to further differentiate between available secondary or tertiary multidisciplinary rehabilitation programmes (figure 2 ).

\section{Integral focus on treatment content and duration: treatment} protocols

When the patient receives treatment, an individualised treatment plan based on their current needs will be made. The patient decides the treatment aim when he visits a healthcare professional. In case this is necessary, the practitioner will support the patient in setting functional goals. Protocols will be based on the most recent evidence-based treatment methods such as GA, EXP and $\mathrm{ACT}^{35-38} 40$ and these will be used in all healthcare settings. As these evidence-based methods are developed for secondary and tertiary care, they will be adjusted for primary care. During evaluations in phases 1 and 2, healthcare professionals will be invited to provide feedback on the treatment protocols. As a result, adjustments to the content and duration of treatment protocols will be 
made if these adjustments are in line with the evidencebased treatment methods.

\section{Integral focus on self-management: E-health application}

All professionals and patients participating in the NPRL will make use of an E-health application: SanaCoach Pain Rehabilitation. ${ }^{55}$ Also, primary care patients who receive 'advice only' can make use of this SanaCoach Pain Rehabilitation. The coach has different functions and goals in the treatment process. The primary goal is to support self-management. The main function of the coach is to provide pain education based on the education modules. Different eLearning modules are developed for the patients in order to teach them about the biopsychosocial aspects of pain. Furthermore, diaries are integrated into the coach in which patients can give feedback on changes in pain intensity, level of activity over time and the inter-relation between these variables. Moreover, healthcare professionals can use scores from these diaries to adjust treatment to individual patients. The coach also consists of a chat function between the patient and healthcare professionals to ensure short communication lines. All healthcare professionals involved in the care process of a patient have access to this chat function with that patient. Additionally, the assessment tool for primary care is integrated, which makes these results available for all involved healthcare professionals. For this study, the questionnaires for patients are also available via the coach. Based on the level of complexity of disability, the functions in the SanaCoach Pain Rehabilitation will be adjusted to the patient, such as the number of diaries and level of education.

\section{Patient and public involvement}

During the development of the research question, design, recruitment and conduct of the study, no patients were involved in the process. However, during the development of NPRL itself, a patient was involved in the development of the SanaCoach Pain Rehabilitation and treatment protocols. Moreover, the focus of this feasibility study is mainly on healthcare professionals. They were involved in the development of the treatment protocols, SanaCoach Pain Rehabilitation and in the development of the different communication strategies between the healthcare professionals themselves. The results of the study will be disseminated to the study participants via the webpage (www.netwerkpijnrevalidatie.nl) and social media accounts.

\section{Data collection}

In this study, the feasibility of the development, implementation and transferability of NPRL for adults with CMP will be investigated. Therefore, different data collection techniques such as observations, interviews, focus groups and questionnaires will be combined to get more insight into the barriers and facilitators of NPRL (table 3).

During the informative meetings and education days, field notes will be made in order to collect information about the views on NPRL and its elements out of the perspectives of the healthcare professionals involved. At the end of each phase, focus groups and/or interviews will take place with (a selection of) the healthcare professionals involved. During the evaluation of phase 1 , healthcare professionals will be asked about the barriers and facilitators they perceived while working in NPRL. Therefore, more information will be collected about expectations, views, experiences and satisfaction. Also, experiences and opinions about the informative meetings and education days will be collected. Healthcare professionals will fill in an electronic questionnaire in phase 1 concerning decision making, treatments and characteristics of the patients involved in the study. This information will give more insight into potential changes in referral policy between the situation in usual care and the situation within NPRL. Moreover, the questionnaire also asks for knowledge and perspectives regarding patients with CMP.

In phases 2 and 3, more emphasis will be put on the added value of NPRL including barriers and facilitators for implementation. This information will be used for recommendations for practise and future research. Also in these phases, information will be gained about the experiences and satisfaction with NPRL during a focus group with healthcare professionals. Moreover, in phase 3 (transferability), they will fill in an electronic questionnaire concerning decision making, treatments and characteristics of the patients involved in the study. As part of the evaluation of phase 3 , a focus group with a sample of 6 to 10 patients with CMP who are being treated by participating healthcare professionals will take place. During this focus group, the emphasis will be on the satisfaction of care and experiences, leading to barriers and facilitators with NPRL.

Besides this information, the research team will keep up a logbook to get insight into the barriers and facilitators of NPRL. The field notes in this logbook will be the results of discussions with different healthcare professionals, patients and stakeholders, as well as researchers. Additionally, patients will be asked to complete study-related questionnaires about the quality and their satisfaction with the decision making, treatment and education and usability of the SanaCoach Pain Rehabilitation in order to further improve different elements of NPRL. Besides this feasibility data, also some questions about their work status, general health and participation level will be asked as preliminary data on efficiency to objectify the progress of the treatment. They will receive this questionnaire at the start of the treatment (T0) and at the end of the treatment (T2). Patients referred to another healthcare professional will receive an extra questionnaire after the referral (T1) regarding the quality of and satisfaction with the decision making. Additionally, after completion of the treatment, a small questionnaire or logbook about the treatment of each patient separately must be handed in by the healthcare professionals. This information will be used to discover barriers and 
Table 3 Overview of data collection methods and respondents per phase

\begin{tabular}{|c|c|c|c|}
\hline Phase & 1 & 2 & 3 \\
\hline Time period & October 2017-February 2018 & February 2018-June 2018 & June 2018-October 2018 \\
\hline Goal project & $\begin{array}{l}\text { Exploration of context will take } \\
\text { place in order to develop the } \\
\text { design of the NPRL and to } \\
\text { educate the involved healthcare }\end{array}$ & $\begin{array}{l}\text { Specification of the content } \\
\text { to adjust the design of the } \\
\text { transmural network to daily } \\
\text { practise. }\end{array}$ & $\begin{array}{l}\text { Organisation of care in daily practise and } \\
\text { barriers and facilitators for implementation in } \\
\text { other practices and organisations. }\end{array}$ \\
\hline
\end{tabular}
educate the involved healthcare practise. professionals.

\begin{tabular}{|c|c|c|c|}
\hline $\begin{array}{l}\text { Goal } \\
\text { evaluation }\end{array}$ & $\begin{array}{l}\text { Insight into the barriers and } \\
\text { facilitators of the development } \\
\text { of NPRL. }\end{array}$ & $\begin{array}{l}\text { Insight into the barriers } \\
\text { and facilitators of the } \\
\text { implementation of NPRL. }\end{array}$ & $\begin{array}{l}\text { Insight into the barriers and facilitators of the } \\
\text { transferability of NPRL. }\end{array}$ \\
\hline $\begin{array}{l}\text { Data collection } \\
\text { method, } \\
\text { respondents } \\
\text { and outcomes }\end{array}$ & $\begin{array}{l}\text { Focus groups and interviews } \\
\text { Healthcare professionals } \\
\text { Experiences with the } \\
\text { informative meetings } \\
\text { Experiences with the } \\
\text { education days } \\
\text { Expectations and views on } \\
\text { working in NPRL } \\
\text { Current experiences } \\
\text { (satisfaction) with working in } \\
\text { NPRL } \\
\text { Barriers and facilitators } \\
\text { Questionnaire } \\
\text { Healthcare professionals } \\
\text { Current views and thoughts } \\
\text { regarding patients with CMP } \\
\text { Referral pattern } \\
\text { Patient characteristics }\end{array}$ & $\begin{array}{l}\text { Focus groups and interviews } \\
\text { Healthcare professionals } \\
\text { Views on working in NPRL } \\
\text { Current experiences } \\
\text { (satisfaction) with working in } \\
\text { NPRL } \\
\text { Implications and } \\
\text { recommendations of the } \\
\text { implementation strategy for } \\
\text { practise } \\
\text { Barriers and facilitators }\end{array}$ & $\begin{array}{l}\text { Focus groups and interviews } \\
\text { Healthcare professionals } \\
\text { Current experiences (satisfaction) with } \\
\text { working in NPRL } \\
\text { Implications and recommendations of the } \\
\text { implementation strategy for practise } \\
\text { - Implications and recommendations for } \\
\text { future research and project } \\
\text { Satisfaction with NPRL and with work life } \\
\text { - Barriers and facilitators } \\
\text { Focus group } \\
\text { +/-10 patients } \\
\text { Perceived quality of care } \\
\text { Experiences with NPRL } \\
\text { Barriers and facilitators } \\
\text { Questionnaire } \\
\text { Healthcare professionals } \\
\text { Referral pattern } \\
\text { Patient characteristics }\end{array}$ \\
\hline & \multicolumn{3}{|c|}{$\begin{array}{l}\text { Questionnaire start and end of treatment (T0 and T2) } \\
\text { Patients } \\
\text { Health status } \\
\text { Quality of care } \\
\text { Questionility of the SanaCoach Pain Rehabilitation } \\
\text { Patients } \\
\text { Quality and satisfaction with referral and care } \\
\text { Questionnaire or logbook of treatment } \\
\text { Healthcare specialists } \\
\text { Botes } \\
\text { Barriers and facilitators of the treatment protocol per patient } \\
\text { Barriers and facilitators }\end{array}$} \\
\hline
\end{tabular}

CMP, chronic musculoskeletal pain; NPRL, Network Pain Rehabilitation Limburg.

facilitators and desired adjustments of the treatment protocols.

\section{Data analysis}

In this iterative design with key principles of user-centred design, the results will be gathered in daily practice from the healthcare professional and patient perspective. The results of each phase will be used to adapt the intervention for the next phase. The CFIR protocol, according to Damschroder et $a l^{53}$ will be used to develop this feasibility evaluation and analysis plan of the results. This explanatory framework with theory-based constructs and mechanisms will be used to explain whether an implementation may or may not succeed and to identify barriers and facilitators.

All field notes and logbooks will be collected. Additionally, the focus groups and interviews will be audio recorded and transcribed verbatim. Qualitative data will be analysed using the NVivo software (NVivo V.11.1.0.411) following a directed content analysis method. ${ }^{59}$ The analysis will be deductive (eg, the identified themes will derive from existing theory). After familiarisation with the data, definitions for the CFIR constructs will be made based on the 
intervention in collaboration with the project team. Next, the different constructs will be assigned to the fewest codes possible. After developing analytic summaries and matrices, the data will be compared with derive barriers and facilitators. A researcher with expertise in qualitative research without any involvement in the project will peer review the analysis by verification of the analysis of $20 \%$ of the interviews and focus groups. Also, a cross-check for interim findings with respondents will be performed.

Quantitative data will be analysed concurrently with the qualitative data. Descriptive statistics will be denoted as mean (SD) or median (range) and number (\%) for continuous and categorical data, respectively, with the use of IBM SPSS Statistics V.24.

\section{ETHICS AND DISSEMINATION}

Informed consent will be obtained from all participants. Ethical approval for this study was granted. The results of this feasibility study will form the base for refinement of NPRL and planning of a large-scale process and effect evaluation on the Quadruple Aim outcomes. Dissemination will include publications and presentations at national and international conferences.

\section{DISCUSSION}

This study will provide insight into the feasibility of NPRL, a transmural integrated healthcare network for CMP rehabilitation. The aim is to provide integrated care for patients with CMP in order to improve their level of functioning despite pain by stimulating a biopsychosocial approach for all involved healthcare professionals. It is expected that the study will provide information on barriers and facilitators, perceived value, acceptability and implementation strategies for the development, implementation and transferability for further develop and refinement of the NPRL. If the study results suggest that NPRL is feasible and preliminary outcomes are positive, a large-scale process and effective evaluation of the Quadruple Aim outcomes will be performed.

The process of developing NPRL is in accordance with the Medical Research Council guidance on how to develop and evaluate complex interventions. ${ }^{52}$ In the development process, existing evidence together with collected evidence based on the expertise of healthcare professionals was combined to develop the first version of NPRL. This first version of NPRL will be implemented on a restricted scale to test the feasibility. The evidence generated from this feasibility study will not only help to adjust the design and content of NPRL but will also inform future methodological studies on developing and implementing a transmural network in healthcare. It is expected that this bottom-up development in combination with the limited number of participating healthcare professionals will lead to a successful implementation of the network. Nijkrake $e t a l^{44}$ did indicate this approach as one of the success factors of ParkinsonNet, a successful and cost-effective network in the Netherlands for patients with Parkinson's disease.

In conclusion, there is need for a transmural network in which different healthcare professionals collaborate in providing integrated healthcare for patients with CMP. The aim of NPRL is to improve the level of functioning of individual patients despite pain, experience of care by patients and work-life satisfaction for physicians and staff, as well as a reduction in costs. Therefore, this feasibility study will be conducted to explore the barriers and facilitators of the development, implementation and transferability of NPRL. The results will be applied to refine a large-scale process and effective evaluation of the Quadruple Aim outcomes.

Acknowledgements We would like to thank Miriam Tabak, as a representative of the patient organisation Pijnpatiënten naar één stem, for her contribution in the development of the SanaCoach Pain Rehabilitation Limburg and the treatment protocols. Moreover, we would like to thank Mario Geilen, physician assistant Adelante Centre of Expertise in Rehabilitation and Audiology, Hoensbroek, The Netherlands, for his contribution in the development of the treatment protocols and the education of the healthcare professionals.

Contributors $\mathrm{JV}, \mathrm{IH}$ and AK conceived the original idea and outline of the study. $\mathrm{CL}, \mathrm{MK}, \mathrm{IH}, \mathrm{JV}$ and DR contributed to designing the study. JV, IH and AK were responsible for developing the intervention. $\mathrm{CL}$ was the primary writer of the study protocol in collaboration with MK and IH. All authors discussed and commented on draft versions and approved the final version.

Funding This work was funded by Health Insurance Companies CZ, VGZ and Achmea, the Netherlands.

Competing interests $\mathrm{AK}, \mathrm{JV}$ and IH report grants from Health Insurance Companies CZ, VGZ and Achmea, during the conduct of the study.

Patient consent for publication Not required.

Ethics approval Approval from the Medical Ethics Committee Z, the Netherlands, METC 17-N-133.

Provenance and peer review Not commissioned; externally peer reviewed.

Open access This is an open access article distributed in accordance with the Creative Commons Attribution Non Commercial (CC BY-NC 4.0) license, which permits others to distribute, remix, adapt, build upon this work non-commercially, and license their derivative works on different terms, provided the original work is properly cited, appropriate credit is given, any changes made indicated, and the use is non-commercial. See: http://creativecommons.org/licenses/by-nc/4.0/.

\section{REFERENCES}

1. Breivik H, Collett B, Ventafridda V, et al. Survey of chronic pain in Europe: prevalence, impact on daily life, and treatment. Eur J Pain 2006;10:287-333.

2. Bekkering GE, Bala MM, Reid K, et al. Epidemiology of chronic pain and its treatment in The Netherlands. Neth J Med 2011;69:141-53.

3. Breivik H, Eisenberg E, O'Brien T, et al. The individual and societal burden of chronic pain in Europe: the case for strategic prioritisation and action to improve knowledge and availability of appropriate care. BMC Public Health 2013;13:1229.

4. Bushnell MC, Ceko M, Low LA. Cognitive and emotional control of pain and its disruption in chronic pain. Nat Rev Neurosci 2013;14:502-11.

5. Gatchel RJ, Peng YB, Peters ML, et al. The biopsychosocial approach to chronic pain: scientific advances and future directions. Psychol Bull 2007;133:581-624.

6. Bair MJ, Robinson RL, Katon W, et al. Depression and pain comorbidity: a literature review. Arch Intern Med 2003;163:2433-45.

7. Jackson T, Wang Y, Wang Y, et al. Self-efficacy and chronic pain outcomes: a meta-analytic review. J Pain 2014;15:800-14.

8. Lerman SF, Rudich Z, Brill S, et al. Longitudinal associations between depression, anxiety, pain, and pain-related disability in chronic pain patients. Psychosom Med 2015;77:333-41.

9. Borghouts J, Janssen $\mathrm{H}$, Koes B, et al. The management of chronic neck pain in general practice. A retrospective study. Scand J Prim Health Care 1999;17:215-20. 
10. Huisstede BM, Wijnhoven HA, Bierma-Zeinstra SM, et al. Prevalence and characteristics of complaints of the arm, neck, and/or shoulder (CANS) in the open population. Clin J Pain 2008;24:253-9.

11. Zorgstandaard Chronische Pijn 2017. 2017. http://www. dutchpainsociety.nl/files/ZorgStandaard_Chronische_Pijn_versie_ 2017_03_28.pdf (accessed 23 Jul 2018).

12. Rijksinstituut voor Volksgezondheid en Milieu. 2018. https://www. volksgezondheidenzorg.info/ (accessed 19 Jul 2018).

13. Niv D, Devor M. European Federation of IASP Chapters. Position paper of the European Federation of IASP Chapters (EFIC) on the subject of pain management. Eur J Pain 2007;11:487-9.

14. Krol M, de Boer D, Plass A, et al. CQ-index module Pijn: meetinstrumentontwikkeling. Utrecht: NIVEL, 2013.

15. Köke AJ, Smeets RJ, Schreurs KM, et al. Dutch Dataset Pain Rehabilitation in daily practice: Content, patient characteristics and reference data. Eur J Pain 2017;21:434-44.

16. Peerenboom PBG, Spek J. Medisch Specialistische Revalidatie; Aanspraak en praktijk. Elst, The Netherlands: Tangram Zorgadviseurs, 2013.

17. Committee on Education of the EFIC (European Federation of IASP Chapters). The Pain Management Core Curriculum for European Medical Schools. 33, 2013.

18. Froud R, Patterson S, Eldridge $S$, et al. A systematic review and meta-synthesis of the impact of low back pain on people's lives. BMC Musculoskelet Disord 2014;15:50.

19. Jellema $\mathrm{P}$, van der Windt DA, van der Horst HE, et al. Why is a treatment aimed at psychosocial factors not effective in patients with (sub)acute low back pain? Pain 2005;118:350-9.

20. Hill JC, Dunn KM, Lewis M, et al. A primary care back pain screening tool: identifying patient subgroups for initial treatment. Arthritis Rheum 2008;59:632-41.

21. Hill JC, Whitehurst DG, Lewis M, et al. Comparison of stratified primary care management for low back pain with current best practice (STarT Back): a randomised controlled trial. Lancet 2011;378:1560-71.

22. Linton SJ, Boersma K. Early identification of patients at risk of developing a persistent back problem: the predictive validity of the Orebro Musculoskeletal Pain Questionnaire. Clin J Pain 2003;19:80-6.

23 van Eijk-Hustings Y, Kroese M, Bessems-Beks M, et al. Supporting healthcare professionals systematically to improve the quality and person-centeredness of fibromyalgia management in primary healthcare. European Journal of Pain 2012;14:2

24. Boonstra AM, Schiphorst Preuper HR, Brouwer M, et al. Classificatie van patiënten met chronische pijnklachten; onderzoek naar de betrouwbaarheid van de WPN niveaus. Nederlandstalig Tijdschrift Pijnbestrijding 2011;30.

25. Waterschoot FP, Bennen E, van der Woude LH, et al. Case complexity in patients with chronic nonspecific musculoskeletal pain a Delphi and feasibility study. Int J Rehabil Res 2016;39:48-56.

26. van Tulder M, Becker A, Bekkering T, et al. Chapter 3. European guidelines for the management of acute nonspecific low back pain in primary care. Eur Spine J 2006;15(Suppl 2):s169-s191.

27. Johnson RE, Jones GT, Wiles NJ, et al. Active exercise, education, and cognitive behavioral therapy for persistent disabling low back pain: a randomized controlled trial. Spine 2007;32:1578-85.

28. Lamb SE, Hansen Z, Lall R, et al. Group cognitive behavioural treatment for low-back pain in primary care: a randomised controlled trial and cost-effectiveness analysis. Lancet 2010;375:916-23.

29. Vibe Fersum K, O'Sullivan P, Skouen JS, et al. Efficacy of classification-based cognitive functional therapy in patients with nonspecific chronic low back pain: a randomized controlled trial. Eur $J$ Pain 2013;17:916-28.

30. Hall A, Richmond H, Copsey B, et al. Physiotherapist-delivered cognitive-behavioural interventions are effective for low back pain, but can they be replicated in clinical practice? A systematic review. Disabil Rehabil 2018:40:1-9.

31. van Erp RMA. Back on Track: Chronic Low Back Pain Rehabilitation in Primary Care [PhD thesis: Maastricht University, 2017.

32. Brage $\mathrm{K}$, Ris I, Falla $\mathrm{D}$, et al. Pain education combined with neckand aerobic training is more effective at relieving chronic neck pain than pain education alone--A preliminary randomized controlled trial. Man Ther 2015;20:686-93.

33. Geneen LJ, Martin DJ, Adams N, et al. Effects of education to facilitate knowledge about chronic pain for adults: a systematic review with meta-analysis. Syst Rev 2015;4:132.
34. Louw A, Diener I, Butler DS, et al. The effect of neuroscience education on pain, disability, anxiety, and stress in chronic musculoskeletal pain. Arch Phys Med Rehabil 2011;92:2041-56.

35. Hayes SC. Acceptance and Commitment Therapy, Relational Frame Theory, and the Third Wave of Behavioral and Cognitive Therapies Republished Article. Behav Ther 2016;47:869-85.

36. Kamper SJ, Apeldoorn AT, Chiarotto A, et al. Multidisciplinary biopsychosocial rehabilitation for chronic low back pain: Cochrane systematic review and meta-analysis. BMJ 2015;350:h444.

37. Lindström I, Ohlund C, Eek C, et al. The effect of graded activity on patients with subacute low back pain: a randomized prospective clinical study with an operant-conditioning behavioral approach. Phys Ther 1992;72:279-90. discussion 91-3.

38. Mansell G, Hall A, Toomey E. Behaviour change and selfmanagement interventions in persistent low back pain. Best Pract Res Clin Rheumatol 2016;30:994-1002.

39. Nicholl BI, Sandal LF, Stochkendahl MJ, et al. Digital support interventions for the self-management of low back pain: a systematic review. J Med Internet Res 2017;19:e179.

40. Vlaeyen JW, Linton SJ. Fear-avoidance model of chronic musculoskeletal pain: 12 years on. Pain 2012;153:1144-7.

41. Zorg RKvan. Chronische pijn. Den Haag 2011.

42. Linton SJ, Nicholas M, Shaw W. Why wait to address high-risk cases of acute low back pain? A comparison of stepped, stratified, and matched care. Pain 2018;159:2437-41.

43. Haarsma F, Moser A, Beckers M, et al. The perceived impact of public involvement in palliative care in a provincial palliative care network in the Netherlands: a qualitative study. Health Expect 2015;18:3186-200.

44. Nijkrake MJ, Keus SH, Overeem S, et al. The ParkinsonNet concept: development, implementation and initial experience. Mov Disord 2010;25:823-9.

45. Rosendal H. Comparative cohort studies in transmural care. Utrecht University: Utrecht, 2002.

46. World Health Organization. Rehabilitation in health systems. Geneva 2017.

47. Krug E, Cieza A. Strengthening health systems to provide rehabilitation services, 2017:95.

48. Berwick DM, Nolan TW, Whittington J. The triple aim: care, health, and cost. Health Aff 2008;27:759-69.

49. Bodenheimer T, Sinsky C. From triple to quadruple aim: care of the patient requires care of the provider. Ann Fam Med 2014:12:573-6.

50. Gulliksen J, Göransson B, Boivie I, et al. Key principles for usercentred systems design. In: Seffah A, Gulliksen J, Desmarais MC, eds. Human-centered software engineering - integrating usability in the software development lifecycle. Dordrecht: Springer Netherlands, 2005:17-36.

51. Hochstenbach LMJ, Courtens AM, Zwakhalen SMG, et al. Cocreative development of an eHealth nursing intervention: Selfmanagement support for outpatients with cancer pain. Appl Nurs Res 2017;36:1-8.

52. Craig P, Dieppe P, Macintyre S, et al. Developing and evaluating complex interventions: the new Medical Research Council guidance. BMJ 2008;337:a1655.

53. Damschroder LJ, Aron DC, Keith RE, et al. Fostering implementation of health services research findings into practice: a consolidated framework for advancing implementation science. Implement Sci 2009;4:50.

54. Leung FH, Savithiri R. Spotlight on focus groups. Can Fam Physician 2009;55:218-9

55. Sananet Care BV. Sananet: specialist in eHealth. http://www.sananet. nl/ehealth-specialist.html (accessed 09 Aug 2018).

56. Leemrijse CJ, Swinkels IC, Veenhof C. Direct access to physical therapy in the Netherlands: results from the first year in communitybased physical therapy. Phys Ther 2008;88:936-46.

57. Huyse FJ, Lyons JS, Stiefel FC, et al. "INTERMED": a method to assess health service needs. I. Development and reliability. Gen Hosp Psychiatry 1999;21:39-48.

58. Stiefel FC, de Jonge P, Huyse FJ, et al. "INTERMED": a method to assess health service needs. II. Results on its validity and clinical use. Gen Hosp Psychiatry 1999;21:49-56.

59. Hsieh HF, Shannon SE. Three approaches to qualitative content analysis. Qual Health Res 2005;15:1277-88.

60. Werkgroep Pijnrevalidatie Nederland. Medisch Specialistische Revalidatie bij chronische pijn aan het houdings- en bewegingsapparaat. Position Paper. Utrecht, 2017. 